\title{
Risk factors for paraaortic lymph-node recurrence in colorectal cancer
}

Shuhei Mayanagi ${ }^{1)}$, Kosuke Kashiwabara ${ }^{2)}$, Michitaka Honda ${ }^{3)}$, Koji Oba ${ }^{4)}$, Toru Aoyama ${ }^{5)}$, Mitsuro Kanda ${ }^{6)}$, Hiromichi Maeda $^{7)}$, Chikuma Hamada ${ }^{8)}$, Sotaro Sadahiro ${ }^{9)}$, Junichi Sakamoto ${ }^{10,11}$, Yoshihiko Maehara ${ }^{11)}$, Takaki Yoshikawa ${ }^{12)}$

1) Department of Surgery, Keio University School of Medicine

${ }^{2)}$ Biostatistics Division, Clinical Research Support Center, The University of Tokyo Hospital

${ }^{3)}$ Department of Minimally Invasive Surgical and Medical Oncology, Fukushima Medical University

${ }^{4)}$ Department of Biostatistics, Graduate School of Medicine, The University of Tokyo

${ }^{5)}$ Department of Surgery, Yokohama City University

${ }^{6)}$ Department of Gastroenterological Surgery (Surgery II), Nagoya University Graduate School of Medicine

${ }^{7)}$ Cancer Treatment Center, Kochi Medical School Hospital

${ }^{8)}$ Faculty of Engineering, Tokyo University of Science

${ }^{9)}$ Department of Surgery, Tokai University

${ }^{10)}$ Tokai Central Hospital

${ }^{11}$ Japanese Foundation for Multidisciplinary Treatment of Cancer

${ }^{12)}$ Department of Gastric Surgery, National Cancer Center Hospital

\begin{abstract}
Background: The paraaortic lymph-node (PALN) is a relatively uncommon metastasis as a first site of recurrence following colorectal cancer (CRC) surgery. Localized and resectable PALN recurrence has the potential of long-term survival by curative resection. We evaluated the risk factors for the recurrence of PALN following curative surgery in patients with CRC in a pooled analysis of two large randomised control studies.

Patients and Methods: Individual patient data from the Japanese Foundation for Multidisciplinary Treatment of Cancer clinical Trials 7 and 15 were pooled for this analysis. We included total 4459 patients who had stage I-III colorectal cancer and underwent curative resection with over $\mathrm{D} 2$ lymph node dissection.

Results: Recurrent PALN occurred in $0.7 \%$ of all patients (30/4459). Of the 30 patients with recurrent PALN, 19 had PALN alone, whereas 11 had a recurrence in at least one other organ in addition to PALN. PALN recurrence occurred after the 3 -year postoperative period in 10 patients (33\%). In multivariate analysis, lymph node involvement was the only independent predictor of recurrent PALN (hazard ratio, 2.670; $\mathrm{p}=0.0106$ ).

Conclusions: Our findings clarify the risk factors for PALN recurrence in stage I-III CRC who undergo curative resection. These results will be useful to identify optimal subgroups for high risk of PLAN recurrence.
\end{abstract}

Keywords: adjuvant chemotherapy, colon cancer, paraaortic lymph node, postoperative recurrence, rectal cancer

(Received September 25, 2019; Accepted October 11, 2019)

\section{INTRODUCTION}

In 2018, colorectal cancer (CRC) was the third most common malignancy in the world accounting for $>1.8$ million cases, i.e. $10 \%$ of total cases, and the second most frequent cause of cancer-associated mortality with 881,000 cases, i.e. $9 \%^{1)}$. In Japan, CRC is a major cause of mortality accounting for the largest number of cases

Correspondence to: Shuhei Mayanagi, Department of Surgery, Keio University School of Medicine, 35 Shinanomachi, Shinjuku-ku, Tokyo 160-8582, Japan TEL: +81-3-3353-1211, Ext. 62334; FAX:+ 81-3-3355-4707 E-mail: s.mayanagi@keio. jp and Takaki Yoshikawa, MD, PhD. Department of Gastric Surgery, National Cancer Center Hospital, 5-1-1 Tsukiji, Chuo-ku, Tokyo 104-0045, Japan TEL: +81-3-35422511 FAX: +81-3-3542-3815 E-mail: tayoshik@ncc.go.jp of cancer-associated mortality among women, and the third largest among men. Although surgical resection is the only curative treatment for CRC, postoperative recurrences often occur in patients who have undergone resection. The typical sites of local recurrence are categorized into the following four groups: the anastomotic site, mesentery or nodal basin, peritoneum and retroperitoneum ${ }^{2}$.

The paraaortic lymph-node (PALN), which is often classified as a non-regional or distant lymph node of the retroperitoneum in patients with $\mathrm{CRC}$, is a relatively uncommon metastasis as a first site of recurrence following surgery. Isolated PALN recurrence was identified in only $1.3 \%$ cases in a previous single institutional retrospective cohort study ${ }^{3)}$. Unlike other gastrointestinal malignancies, localized and resectable PALN recurrence has the 
potential of long-term survival following curative resection. However, the recurrence of PALN is often accompanied by metastasis to other organs. As in the case of any recurrence, early detection is necessary to allow treatment of the recurrent tumour. Therefore, it is important to clarify the risk factors for these recurrences. However, there is no previous report regarding the risk factors for the recurrence of PALN. In this study, we evaluated the risk factors for the recurrence of PALN following curative surgery in patients with CRC in a pooled analysis of two large randomised control studies.

\section{PATIENTS AND METHODS}

\section{Patients}

Individual patient data from the Japanese Foundation for Multidisciplinary Treatment of Cancer (JFMC) clinical Trials 7 and 15 were pooled for this analysis ${ }^{4)}$.

\section{JFMC Trials 7 and 15}

In JFMC Trials 7 and 15, patients with locally advanced $\mathrm{CRC}$ were randomly allocated either to receive adjuvant chemotherapy with oral 5-fluorouracil (FU) or surgery alone ${ }^{5)}$. In the Japanese Classification of Colorectal Carcinoma, regional lymph nodes are classified into the following three groups according to their position: D1 (pericolic) nodes are situated close to the bowel wall, D2 (intermediate) nodes lie along the feeding arteries and D3 (main) nodes are located at the origin of the feeding artery ${ }^{6}$. In these Trials, curative resection with over D2 lymph node dissection was performed. The study design was similar for both Trials. The main inclusion criteria were as follows: 1) macroscopic Dukes' B (invasion through the bowel wall penetrating the muscle layer but not involving lymph nodes) or Dukes' $\mathrm{C}$ (involvement of lymph nodes) based on intraoperative judgement; 2) age < 75 years and 3) no severe complications. Between 1986 and 1990, the total of number of patients enrolled for JFMC 7 and 15 were 3394 and 2315, respectively. The adjuvant chemotherapy comprised a 1-year administration of oral 5-FUs (JFMC 7, 200 or 300 mg/day; JFMC 15, 300 mg/day).

In the present pooled analysis, we included patients who had stage I-III CRC and underwent curative resection with over D2 lymph node dissection. A total of 4459 patients were included in the present study (Fig. 1). The key findings of each Trial have been published in peerreviewed journals.

\section{Follow-up}

Following completion of the treatment protocol, patients were followed-up according to a schedule defined by each clinical trial protocol until recurrence or death for 5 years following surgery. Recurrence was briefly assessed based on computed tomography scans that were performed every 4 months during the first 2 years following surgery, and once every 6 months from the third year onward. The recurrence-free survival was determined from the date of surgery to the date of recurrence for each patient. Recurrences due to other causes, patient succumbing to mortality or survival without recurrence were treated by censoring. The survival from recurrence was determined from the date of recurrence to the date at which the patient succumbed to mortality from any cause. All follow-ups were censored at 5 years from the date of surgery.

This study was performed in accordance with ethical principles having their origins in the Declaration of Helsinki, and approved by the institutional review board of JFMC.

\section{Statistical analysis}

Baseline clinical and pathological variables are expressed as the mean \pm standard deviation for continuous variables or numbers and proportions for categorical variables. For the primary analysis of PALN recurrencefree survival, we applied competing risk analysis, classifying all other recurrences, without PALN recurrence

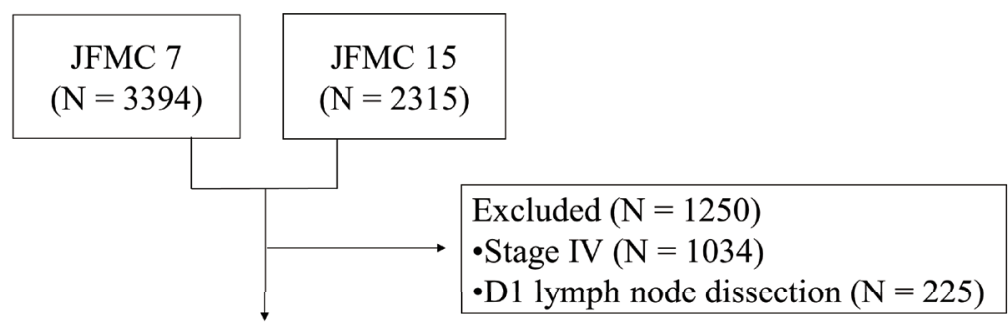

Analysis object $(\mathrm{N}=4459)$

-Stage I / II / III colorectal cancer

- Curative resection with over D2 lymph node dissection

Fig. 1 Flow diagram

We included patients who had stage I-III colorectal cancer and underwent curative resection with over D2 lymph node dissection. In total, 4459 patients were included in the present study. 
or mortality, as competing events using the method described by Fine and Gray $^{7)}$. In our multivariate analysis, because the number of patients with PALN was insufficient for analysis with many predictors, we restricted the predictors a priori to the following four prognostic factors whose association with PALN recurrence was clinically or biologically expected: lymphatic invasion, lymph node involvement, lymphadenectomy and adjuvant chemotherapy. The Kaplan-Meier curve of the survival from recurrence by recurrence sites (PALN/not PALN) was depicted using Kaplan-Meier method, and survival from recurrence was compared between patients with and without PALN using log-rank test. In all analyses, a value of $\mathrm{p}<0.05$ was considered statistically significant. All analyses were performed using SPSS Version 19 software (IBM Corp., Armonk, NY, USA) and SAS version 9.4 (SAS Institute Inc., Cary, NC, USA).

\section{RESULTS}

The clinicopathological parameters of the patients in the pooled analysis are shown in Table 1 . The total cohort consisted of patients with colon cancer $(55 \%)$ and rectal cancer including rectosigmoid (45\%). Tumour depth was pT3 or pT4 in the majority of patients, lymph node involvement was observed in a third of patients and FU-based adjuvant chemotherapy was administered in $57 \%$ of this cohort. Recurrent PALN occurred in $0.7 \%$ of all patients (30/4459). Of the 30 patients with recurrent PALN, 19 had PALN alone, whereas 11 had a recurrence in at least one other organ in addition to PALN. The other organs observed along with PALN were the liver or lung in eight patients and other lymph nodes in three patients. PALN recurrence occurred within the 3-year postoperative period in 20 patients $(67 \%)$ and after the 3 -year postoperative period in 10 patients (33\%).

Following recurrence, treatment for PALN included surgery (11 patients, 36\%), radiotherapy $(4,13 \%)$ and systemic chemotherapy $(23,76 \%)$. The survival from recurrence was lower in patients with recurrent PALN than those with recurrence in the liver or lung $(\mathrm{p}<0.001$; Fig. 2)

In multivariate analysis, lymph node involvement was the only independent predictor of recurrent PALN (hazard ratio, 2.670; $p=0.0106$; Table 2). However, lymphatic invasion, extent of lymphadenectomy and use of adjuvant chemotherapy were not significant factors for predicting the recurrence of PALN.

\section{DISCUSSION}

The present study demonstrated the risk factors for recurrent PALN in patient with stage I-III CRC from pooled individual patient data of two large phase III studies. To the best of our knowledge, this is the first report demonstrating that lymph node involvement is associated with PALN recurrence in patients who undergo curative resection for CRC in multivariate analysis. These results are useful to identify optimal subgroups for high risk of PALN recurrence.

We found the incidence of recurrent PALN to be $0.7 \%$ in the entire patient cohort, which was comparable with the incidence reported in a previous retrospective study ${ }^{3)}$. However, focusing on patients positive for regional lymph node involvement, PALN recurrence was reported to occur more frequently $(13 / 1033,1.25 \%)^{8)}$. Min et al showed that recurrent cases of PALN had metastatic regional lymph nodes in $33 / 38$ cases ( $87 \%$ ), and 28 of those 33 cases were diagnosed with pathological N2 ( $\geq 4$ positive lymph nodes). These reports are compatible with our results and confirm that lymph node involvement is the most important risk factor for PALN recurrence.

It has been reported that the survival rates of patients with complete resection for recurrent PALN are significantly higher than those who do not undergo resec$\operatorname{tion}^{3,9,10)}$, although standard therapy for recurrent PALN has not been esTablished. In addition, aggressive surgical treatment of PALN recurrence, including the concomitant resection of major retroperitoneal vessels, has acceptable morbidity and may be associated with improved survival rate ${ }^{11)}$. Therefore, in patients with high risk for recurrent PALN, the early detection of postoperative PALN recurrence may be important to improve survival. However, as recurrent PALN is often accompanied with other organ metastases, comprising $37 \%$ of all cases of PALN recurrence in the present study, it is important that a radical surgical approach for recurrent PALN is indicated in well-selected patients.

Previous reports on recurrences following curative resection in Europe and the United States have shown that $\sim 70 \%$ of the recurrences are detected within 2 years following surgery ${ }^{12)}$. It has also been reported that $>80 \%$ of the recurrences are detected within 3 years following surgery in Japan ${ }^{13)}$. Interestingly, in our cohort, one third of the patients with PALN had relapsed after 3 years. Previous studies have also reported that the mean time from primary cancer resection to detection of PALN is $>30$ months $^{11,14-16)}$. Therefore, it is necessary for positive cases of lymph node metastasis to be followed-up to 5 years, and careful follow-up would still be required after $\geq 3$ years following surgery.

Although adjuvant chemotherapy is an important factor to prevent any recurrence, it was not associated with the recurrence of PALN in our study. The adjuvant chemotherapy regimens with oral 5-FU were different from current standard regimen because the Trials were performed in the 1980s. Therefore, further investigations are required to clarify the correlation between adjuvant chemotherapy and the recurrence of PALN.

In summary, our findings clarify the risk factors for 
Table 1 Patients' clinicopathological parameters

\begin{tabular}{|c|c|c|c|c|}
\hline & \multicolumn{2}{|c|}{$\begin{array}{c}\text { All } \\
N=4459\end{array}$} & \multicolumn{2}{|c|}{$\begin{array}{c}\text { Recurrent paraaor } \\
\text { tic lymph-node } \\
\mathbf{N}=\mathbf{3 0}\end{array}$} \\
\hline Age, $y$ & \multicolumn{2}{|c|}{$58.5 \pm 9.2$} & \multicolumn{2}{|c|}{$59.9 \pm 7.1$} \\
\hline Sex (male/female) & \multicolumn{2}{|c|}{$\begin{array}{l}2546 / 1913 \\
(57 \% / 43 \%)\end{array}$} & \multicolumn{2}{|c|}{$\begin{array}{c}16 / 14 \\
(53 \% / 47 \%)\end{array}$} \\
\hline \multicolumn{5}{|l|}{ Location } \\
\hline Right colon & 1033 & $23 \%$ & 12 & $40 \%$ \\
\hline Left colon & 1423 & $32 \%$ & 4 & $13 \%$ \\
\hline Rectum & 2001 & $45 \%$ & 14 & $33 \%$ \\
\hline Unknown & 2 & $0 \%$ & 0 & $0 \%$ \\
\hline \multicolumn{5}{|l|}{ pT factor } \\
\hline pT1 & 87 & $2 \%$ & 0 & $0 \%$ \\
\hline pT2 & 639 & $14 \%$ & 4 & $13 \%$ \\
\hline pT3 & 2358 & $53 \%$ & 11 & $37 \%$ \\
\hline pT4 & 1354 & $30 \%$ & 15 & $50 \%$ \\
\hline Unknown & 21 & $1 \%$ & 0 & $0 \%$ \\
\hline \multicolumn{5}{|l|}{$\mathrm{pN}$ factor } \\
\hline Negative & 3002 & $67 \%$ & 13 & $43 \%$ \\
\hline $\mathrm{pN} 1$ & 1434 & $32 \%$ & 17 & $57 \%$ \\
\hline $\mathrm{pN} 2$ & 1 & $0 \%$ & 0 & $0 \%$ \\
\hline Unknown & 22 & $0 \%$ & 0 & $0 \%$ \\
\hline \multicolumn{5}{|l|}{ Resection margin } \\
\hline Positive (R1) & 34 & $1 \%$ & 1 & $3 \%$ \\
\hline \multicolumn{5}{|l|}{ Adjuvant chemotherapy } \\
\hline No & 1921 & $43 \%$ & 11 & $37 \%$ \\
\hline Yes & 2538 & $57 \%$ & 19 & $63 \%$ \\
\hline \multicolumn{5}{|l|}{ Lymphadenectomy } \\
\hline D2 & 2010 & $45 \%$ & 13 & $43 \%$ \\
\hline D3 & 2439 & $55 \%$ & 17 & $57 \%$ \\
\hline Unknown & 10 & $0 \%$ & 0 & $0 \%$ \\
\hline \multicolumn{5}{|l|}{ Histological type } \\
\hline Well and moderate & 4207 & $94 \%$ & 27 & $90 \%$ \\
\hline Poorly & 97 & $2 \%$ & 0 & $0 \%$ \\
\hline Mucinous & 118 & $3 \%$ & 3 & $10 \%$ \\
\hline Others & 37 & $1 \%$ & 0 & $0 \%$ \\
\hline \multicolumn{5}{|l|}{ Lymphatic invasion } \\
\hline Ly(-) & 1574 & $35 \%$ & 9 & $30 \%$ \\
\hline $\mathrm{Ly}(+)$ & 2802 & $63 \%$ & 21 & $70 \%$ \\
\hline Unknown & 83 & $2 \%$ & 0 & $0 \%$ \\
\hline \multicolumn{5}{|l|}{ Venous invasion } \\
\hline $\mathrm{V}(-)$ & 2735 & $61 \%$ & 19 & $63 \%$ \\
\hline $\mathrm{V}(+)$ & 1559 & $35 \%$ & 10 & $33 \%$ \\
\hline Unknown & 165 & $4 \%$ & 1 & $3 \%$ \\
\hline
\end{tabular}

recurrence of PALN in patients with stage I-III CRC who undergo curative resection. These results will be useful to identify optimal subgroups for high risk of PALN recurrence.

\section{ACKNOWLEDGMENT:}

This study was designed and organized by JFMC Manuscript Writing Committee, and supported in part by ECRIN.

\section{CONFLICTS OF INTEREST:}

The authors declare no conflict of interest.

\section{SOURCE OF FUNDING:}

This study was supported by JFMC, and in part, by the Epidemiological and Clinical Research Information Network (ECRIN).
Table 2 Multivariate Cox-regression analysis for paraaortic lymphnode recurrence

\begin{tabular}{l|c|c|c}
\hline & HR & p-value & $95 \%$ CI \\
\hline $\begin{array}{l}\text { Lymphatic invasion } \\
\text { Ly(-) }\end{array}$ & 1 & & \\
$\quad$ Ly(+) & 1.09 & 0.838 & $0.48-2.47$ \\
pN factor & 1 & & \\
$\quad \begin{array}{l}\text { Negative } \\
\text { Positive }\end{array}$ & 2.67 & 0.011 & $1.26-5.67$ \\
Lymphadenectomy & 1 & & \\
$\quad \begin{array}{l}\text { D3 } \\
\text { D2 }\end{array}$ & 0.90 & 0.772 & $0.44-1.85$ \\
$\begin{array}{l}\text { 5-FU based adjuvant chemotherapy } \\
(-)\end{array}$ & 1 & & \\
$\quad(+)$ & 1.45 & 0.350 & $0.66-3.18$ \\
\hline
\end{tabular}

HR hazard ratio, CI confidence interval

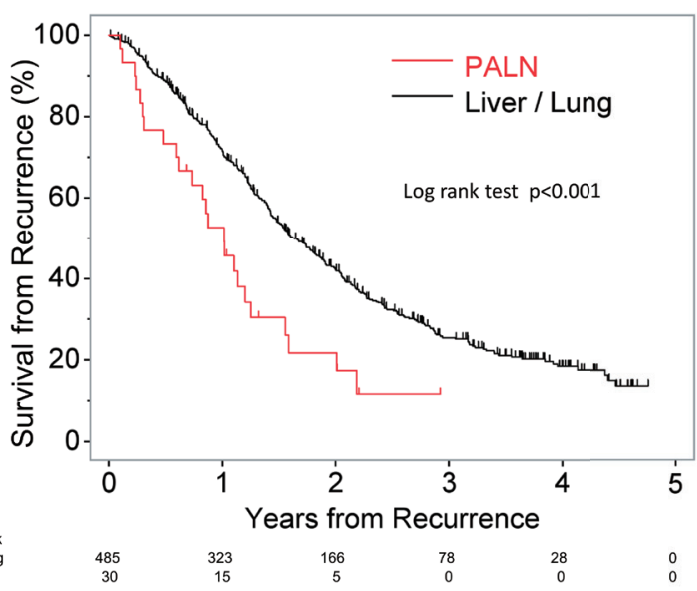

Fig. 2 The Kaplan-Meier graph of survival from recurrence The survival from recurrence is lower among patients with recurrent PALN than among those with liver or lung $(p<0.001)$.

\section{REFERENCES}

1) Bray F, Ferlay J, Soerjomataram I, Siegel RL, Torre LA, Jemal A (2018) Global cancer statistics 2018: GLOBOCAN estimates of incidence and mortality worldwide for 36 cancers in 185 countries. CA Cancer J Clin. DOI: 10.3322/caac.21492

2) Galandiuk S, Wieand HS, Moertel CG, Cha SS, Fitzgibbons RJ Jr, Pemberton JH, Wolff BG (1992) Patterns of recurrence after curative resection of carcinoma of the colon and rectum. Surg Gynecol Obstet 174: 27-32.

3) Min BS, Kim NK, Sohn SK, Cho CH, Lee KY, Baik SH (2008) Isolated paraaortic lymph-node recurrence after the curative resection of colorectal carcinoma. J Surg Oncol 97: 136-140.

4) Mayanagi S, Oba K, Hamada C, Sadahiro S, Kanda M, Maeda H, Aoyama T, Kashiwabara K, Sakamoto J, Saji S, Yoshikawa T (2016) Risk factors associated with recurrence by peritoneal dissemination or paraaortic lymph node metastasis after curative surgery in patients with colorectal cancer. Ann Cancer Res Ther 24: 58-59.

5) Hamada C, Sakamoto J, Satoh T, et al (2011) Does 1 year adjuvant chemotherapy with oral 5-FUs in colon cancer reduce the peak of recurrence in 1 year and provide long-term OS benefit? Jpn J Clin Oncol 41: 299-302. 
6) Japanese Society for Cancer of the Colon and Rectum (2009) Japanese Classification of Colorectal Carcinoma, 2nd English Edition. Kanehara \& Co, Ltd, Tokyo.

7) Fine JP, Gray RJ (1999) A Proportional Hazards Model for the Subdistribution of a Competing Risk. J Am Stat Assoc 94: 496-509.

8) Bae SU, Han YD, Cho MS, Hur H, Min BS, Lee KY, Kim NK (2016) Oncologic Outcomes of Colon Cancer Patients with Extraregional Lymph Node Metastasis: Comparison of Isolated Paraaortic Lymph Node Metastasis with Resectable Liver Metastasis. Ann Surg Oncol 23: 1562-1568.

9) Shibata D, Paty PB, Guillem JG, Wong WD, Cohen AM (2002) Surgical management of isolated retroperitoneal recurrences of colorectal carcinoma. Dis Colon Rectum 45: 795-801.

10) Taylor WE, Donohue JH, Gunderson LL, Nelson H, Nagorney DM, Devine RM, Haddock MG, Larson DR, Rubin J, O'Connell MJ (2002) The mayo clinic experience with multimodality treatment of locally advanced or recurrent colon cancer. Ann Surg Oncol 9: 177185.

11) Ho TW, Mack LA, Temple WJ (2011) Operative salvage for retroperitoneal nodal recurrence in colorectal cancer: a systematic review. Ann Surg Oncol 18: 697-703.

12) Bruinvels DJ, Stiggelbout AM, Kievit J, Dik J, Habbema F, Van de Velde CJ (1994) Follow-up of patients with colorectal cancer. A meta-analysis. Ann Surg 219: 174-182.
13) Watanabe T, Muro K, Ajioka Y, Hashiguchi Y, Ito Y, Saito Y, Hamaguchi T, Ishida H, Ishiguro M, Ishihara S, Kanemitsu Y, Kawano H, Kinugasa Y, Kokudo N, Murofushi K, Nakajima T, Oka S, Sakai Y, Tsuji A, Uehara K, Ueno H, Yamazaki K, Yoshida M, Yoshino T, Boku N, Fujimori T, Itabashi M, Koinuma N, Morita T, Nishimura G, Sakata Y, Shimada Y, Takahashi K, Tanaka S, Tsuruta O, Yamaguchi T, Yamaguchi N, Tanaka T, Kotake K, Sugihara K; Japanese Society for Cancer of the Colon and Rectum (2018) Japanese Society for Cancer of the Colon and Rectum (JSCCR) guidelines 2016 for the treatment of colorectal cancer. Int J Clin Oncol 23: 1-34.

14) Mixter CG Jr. (1980) Resection of the abdominal aorta for recurrent colon cancers. 18 year survival. Am J Surg 139: 595-601.

15) Hashimoto M, Komatsu H, Naruse Y, Sawada T, Watanabe G (2003) Resection of paraaortic lymph node metastasis of colon cancer with graft replacement. Hepatogastroenterology 50: 709-10.

16) Tsarkov PV, Belov YV, Skipenko OG, Zavenyan ZS, Makeev YN, Troitskiy AA, Tulina IA, Bashankaev BN (2007) En bloc resection of abdominal aorta and paraaortic lymph node metastasis of sigmoid cancer. Tech in coloproctol 11: 346-9. 